Mon. Not. R. Astron. Soc. 000, 18 (2007) Printed 20 November 2018 (MN LATEX style file v2.2)

\title{
A panoramic view of M81: New stellar systems in the debris field
}

\author{
M. Mouhcine ${ }^{1}$, R. Ibata ${ }^{2}$ \\ ${ }^{1}$ Astrophysics Research Institute, Liverpool John Moores University, Twelve Quays House, Egerton Wharf, Birkenhead, CH41 1LD, UK \\ ${ }^{2}$ Observatoire Astronomique de Strasbourg (UMR 7550), 11, rue de l'Université, 67000 Strasbourg, France
}

Accepted ?. Received ?; in original form ?

\begin{abstract}
Using the MegaCam imager on the Canada-France-Hawaii Telescope, we have resolved individual stars in the outskirts of the nearby large spiral galaxy M81 (NGC 3031) well below the tip of the red giant branch of metal-poor stellar populations over $\sim 60 \mathrm{kpc} \times 58 \mathrm{kpc}$. In this paper, we report the discovery of new young stellar systems in the outskirts of M81. The most prominent feature is a chain of clumps of young stars distributed along the extended southern Hi tidal arm connecting M 81 and NGC 3077. The colour-magnitude diagrams of these stellar systems show plumes of bright main sequence stars and red supergiant stars, indicating extended events of star formation. The main sequence turn-offs of the youngest stars in the systems are consistent with ages of $\sim 40$ Myr. The newly reported stellar systems show strong similarities with other known young stellar systems in the debris field around M81, with their properties best explained by these systems being of tidal origin.
\end{abstract}

Key words: galaxies: formation - galaxies: stellar content - galaxies: individual (M81, NGC 3077, Holmberg IX, BK 3N, Garland) - galaxies: photometry

\section{INTRODUCTION}

In recent years, it has been increasingly recognised that the outskirts of galaxies hold fundamental clues about their formation history. It is into these regions that new material continues to arrive as part of their assembly, by accretion of minor satellites, predominantly at early epochs when large disk galaxies were assembling, as predicted by the currently favored hierarchical formation models. It was also in the outer regions of galaxies that material was deposited during the violent interations in the galaxy's past. Most present-day disk galaxies are suspected to have experienced mergers during the last few billions of years (e.g. Hammer et al. 2007, and references therein). Based on the systematic deviation of the Milky Way from a number of galaxy scaling relations, Hammer et al. (2007) have agrued that our galaxy had most likely escaped any significant major merger event over the last $\sim 10$ Gyr. These authors suspect that the ob-

1 This publication is based on observations with the MegaPrime/MegaCam, a joint project of the CFHT and CEA/DAPNIA, at the Canada-France-HAwaii Telescope (CFHT), which is operated by the National Research Council of Canada, the institut National des Sciences de l'Univers of the Centre National de la Recherche Scientifique (CNRS), and the University of Hawaii. served differences between the Milky Way and its neighbour M31 are likely due to the quiescent formation in the former case and to the merger-dominated history for the latter. The observed properties of the stellar content in the outskirts of M31 can be accounted for by either a succession of minor mergers or a major merger, with this material most likely accreting in the most recent half of the age of the Universe (Ibata et al. 2005).

By analysing the characteristics of spiral galaxy stellar halos formed within a large grid of numerical chemodynamical simulations, Renda et al. (2005) have shown that at any given total galactic mass, the metallicities of simulated stellar halos span a range in excess of $\sim 1$ dex. The underlying driver of this metallicity spread can be traced back to the diversity of galactic mass assembly histories. Galaxies with a more extended merging history possess halos which have younger and more metal rich stellar populations than the stellar halos associated with galaxies with a more abbreviated assembly. For a given total mass, galaxies with more extended assembly histories also possess more massive stellar halos.

The studies of the Galaxy, and to lesser extent the other large spiral in the Local Group, M31, have been delivering the bulk of the observational constraints on the properties of the stellar content of the outer regions of galaxies. Evidence indicates that the Galaxy might be unrepresentative of a 
typical spiral galaxy, and it may not even follow the standard scenario of disk formation. The Milky Way halo seems to be populated by old, metal-poor stars, while a few fields in the halo of M31 show a large population of intermediate age stars with a much higher overall metallicity Brown et al. 2006). The fields in M31 in which the above results were obtained have been found to be significantly contaminated by various accretion events (Ibata et al. 2007) casting doubt on the conclusion that the M31 halo is globally younger and more metal-rich than that of the Milky Way. The current observational evidence therefore demonstrates that halos are complex structures. To establish comprehensively properties of stars in the outskirts of galaxies, and to fully understand their nature and origin, we need to undertake panoramic studies of the outer regions of spiral galaxies beyond the Local Group. To do so, we have obtained deep and widefield optical imaging data of the nearby early-type spiral M81 (NGC 3031), resolving stars well below the tip of the red giant branch of metal-poor stellar populations.

The M81 group of galaxies is one of the nearest groups to our own. It contains one large spiral, two peculiar galaxies (M82 and NGC 3077), two small spirals galaxies (NGC 2976 and IC 2574), as well as a large number of dwarf galaxies (e.g. Karachentsev et al. 1985, 2001; Chiboucas et al. 2008). The core galaxies of the group are strongly interacting. Atomic hydrogen observations have revealed the presence of a large number of tidal streams with large, dynamically complex atomic hydrogen clouds embedding M81, M82, NGC 3077, and NGC 2976 (van der Hulst 1979; Appleton et al. 1981; Yun et al. 1994; Boyce et al. 2001). Close interactions between galaxies are capable of leaving tidal debris that could be converted into new stellar systems (e.g. Toomre \& Toomre 1972). Compared to the Local Group, an interesting feature of the M81 group is the presence of a population of stellar systems dominated by young stars (e.g. Durrell et al. 2004; de Mello et al. 2008a; Davidge 2008), which are suspected to be of tidal origin (e.g Makarova et al. 2002), and which have no counterparts in the Local Group. These young stellar systems, e.g. Holmberg IX, BK 3N, and Garland, are embedded in Hi clouds (Bovce et al. 2001). Here, we take advantage of our deep and wide field survey to study the spatial distribution of young stellar populations in the outer regions of M81. We report the discovery of new stellar systems in the tidal debris.

Analysis of the spatial distribution of old stellar populations, the bi-dimensional distribution, the search for substructures, the metallicity distribution functions, and globular cluster properties over the surveyed area will be reported in forthcoming papers. The stellar populations of immediate interest to the present paper are revealed by the upper main sequence and the red supergiant stars. The layout of this paper is as follows: in Section 2 briefly represents the data set, while section 3 studies the young stellar content around M 81.

\section{DATA}

The MegaCam wide-field imager at the Canada-FranceHawaii Telescope (CFHT) was used to map M81 over the area displayed in Figure 1. MegaCam consists of a mosaic of

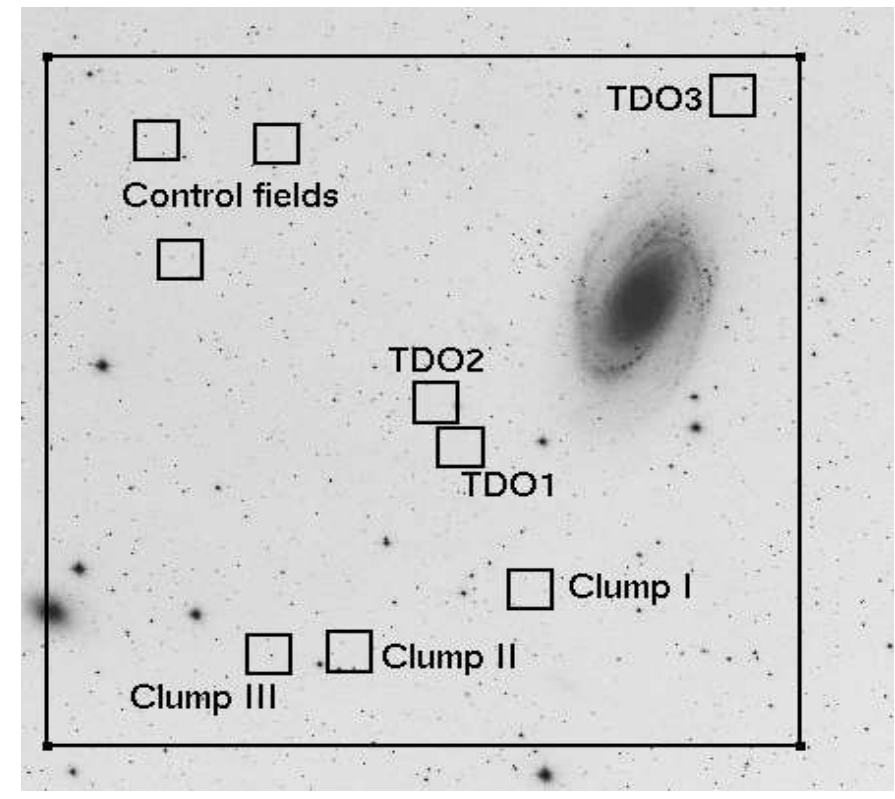

Figure 1. CFHT/MegaCam footprint of the observations overlayed on the Digitized Sky Survey image of M 81. The MegaCam field-of-view covers a $0.96^{\circ} \times 0.94^{\circ}$ field. The three new young stellar clumps reported here are identified as Clump I, II, III (see text for more details). Also shown are the three tidal debris objects (TDO1, TDO2, TDO3) identified by Davidge (2008). The locations of three control fields are also shown.

thirty six $2048 \times 4612 \mathrm{EEV}$ chips, covering a $0.96^{\circ} \times 0.94^{\circ}$ field, with each pixel subtending 0.187 arcseconds on a side. The photometric depth and field of view achievable with this instrument make it particularly powerful in regions of extremely low stellar surface density. To observe as much of the galaxy halo and the extension of the galaxy disk as possible, we positioned the centre of M81 in one corner of the mosaic. The images used in this study are of a single pointing centred at R.A. $=09^{\mathrm{h}} 58^{\mathrm{m}} 44.0^{\mathrm{s}}$, Dec. $=+68^{\circ} 51^{\prime} 46.0^{\prime \prime}$ (J2000). The survey gives an uninterrupted coverage out to approximately $50 \mathrm{kpc}$ along the major axis, and the inner halo out to $\sim 45 \mathrm{kpc}$.

The observations were taken with the $g$ and $i$ filters, with total exposure times of 14000 and 20000 seconds, respectively, in each of these two bands, to reach $g \simeq 27.3$ and $i \simeq 25.9$. At the distance of M81, taken to be 3.55 Mpc (Freedman et al. 1994) throughout this paper, we detect approximately the top 1.5 magnitudes of the red giant branch of metal-poor stellar populations. The data were obtained in dark skies, with typical seeing of 0.9 and 0.8 arcseconds in the g- and i-bands, respectively (the relatively poor seeing is due to the low elevation of the target as observed from Hawaii). The individual exposures were recorded with a square-shaped dither pattern to assist with the identification of bad pixels and the suppression of cosmic rays.

The images were pre-processed by the CFHT Elixir pipeline for corrections for bias, flat-fielding, and the fringing pattern. Photometric standards observed over the season are used to determine the photometric zero point in each passband. The images were then processed by the Cambridge Astronomical Survey Unit (CASU) photometry pipeline (Irwin \& Lewis 2001), in an identical manner to that described in Ségall et al. (2007). The interested reader 

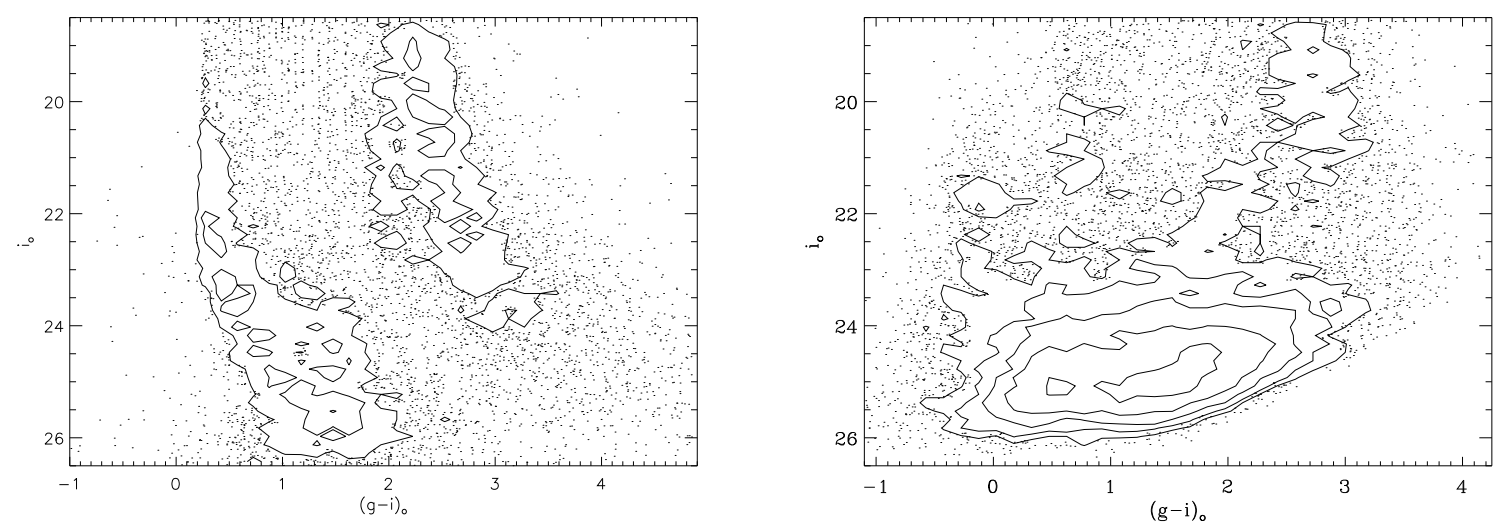

Figure 2. Left: The $i_{\circ}$ versus $(g-i)$ 。CMD of foreground stars as predicted by The Besançon Galactic population model in the direction of M 81 over the same field-of-view as covered with a single MegaCam pointing. Right: The $i_{\circ}$ versus $(g-i) \circ$ CMD of objects detected in our field and classified as stars. Regions of density less than ten stars per bin of $0.15 \times 0.15$ magnitudes are plotted as points. Contours are spaced by factors of two.

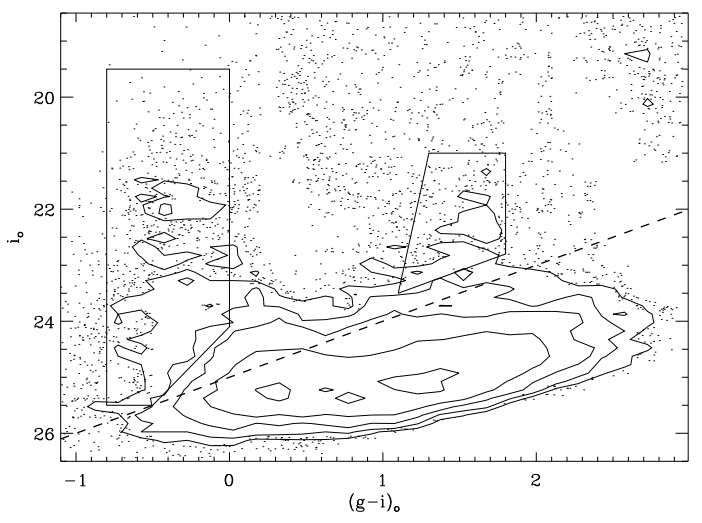

Figure 3. Foreground-corrected CMD of stars in the MegaCam pointing. The solid boxes mark the locations of the colourmagnitude diagram where blue main sequence and red supergiant star candidates lie respectively.

is referred to this paper for more details. The software then proceeds to detect sources and measures their photometry, the image profile, and shape. Based on the information contained in the curve of growth, the algorithm classifies the objects into noise detections, galaxies, and probable stars. Throughout the paper we select as stars objects that have classification of -1 and -2 in both bands. This corresponds to stars up to $2 \sigma$ from the stellar locus. To correct for the foreground extinction, we used the Schlegel et al. (1998) dust map value of $E_{(B=V)}=0.08$, corresponding to $A_{g}=0.303$ and $A_{i}=0.167$ respectively.

\section{RESULTS}

As well as encompassing a large fraction of M81 and its outskirts, the survey also intersects a non-negligible volume of the foreground Galaxy. To subtract off the foreground counts, we used the Besançon Galactic population model (Robin et al. 2003) to predict the foreground contamina-

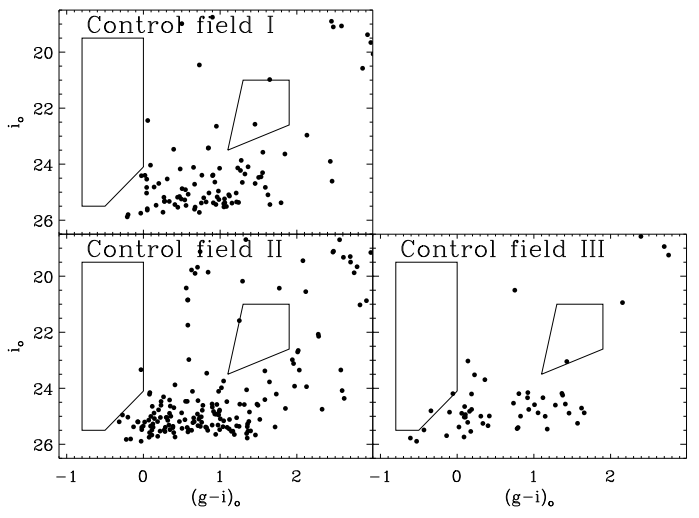

Figure 5. Foreground-corrected CMD of stars in the three control fields. The solid boxes mark the locations of the colourmagnitude diagram where blue main sequence and red supergiant star candidates lie respectively.

tion. This Galaxy model has shown its capacity to predict reliably the observed foreground counts over large areas (see, e.g. Ibata et al. 2007). To substract the background counts, we tessellated the survey area with $0.25^{\circ} \times 0.25^{\circ}$ bins and generated simulated catalogues using the Besançon model. All stellar populations in the models with $i$-band magnitudes between $17<i_{\circ}<26$ were accepted. To reduce noise in the randomly generated catalogues, at each spatial bin we simulated a 30 times larger solid angle and later corrected the density maps for this factor. Finally, the artificial photometry was convolved with the observed magnitude-dependent error function. The left panel of Fig. 2 shows the predicted $i$ 。 vs. $(g-i)$ 。 colour-magnitude diagram (hereafter CMD) of the Galaxy foreground stars in the direction of M81. Regions of the CMDs with stellar densities higher than ten stars in a $0.15 \times 0.15$ magnitude bin are shown as contours with the contour levels spaced uniformly by a factor of two. Stars are plotted as points when the densities are lower. The foreground contamination is dominated by a prominent blue sequence, at $0 \lesssim(g-i)_{\circ} \lesssim 0.8$, of halo stars at or close to 

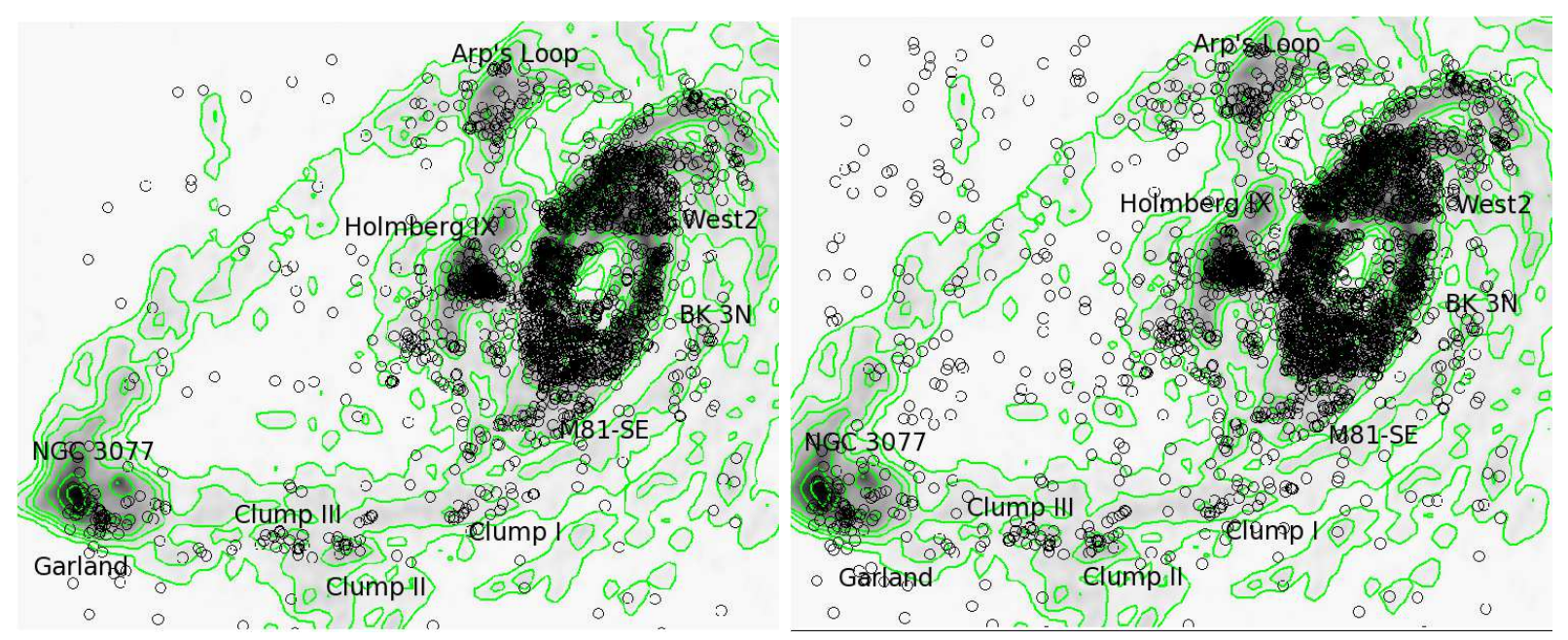

Figure 4. (Left) Spatial distribution of main sequence star candidates, shown as open circles, over the MegaCam pointing. The contours and grayscale image show the Hi radio map from Yun et al. (1994). (Right) similar to the left panel, but for both main sequence and red supergiant star candidates. The Hi image adopted from Yun et al.'s Figure 1 by permission from Macmillan Publishers Ltd: Nature, copyright (1994).

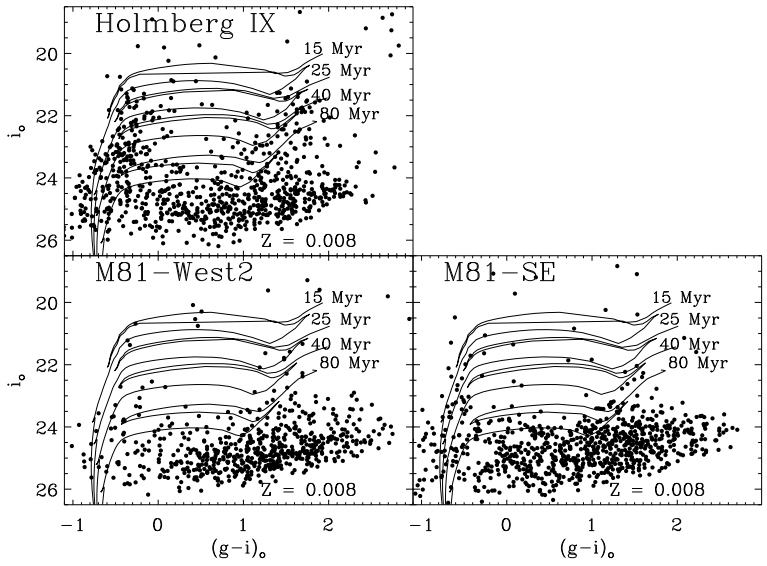

Figure 6. The CMDs of stellar populations tracing the continuations of both the nourthen and the southern Hi spiral arms as identified in Fig.4 Superimposed on each CMD are the Padova isochrones with a metallicity $\mathrm{Z}=0.008$ and the indicated ages. For a comparison purpose, the CMD of Holmberg IX is shown in the upper left panel.

the main sequence turn-off at increasing distance through the Galactic halo. On the red side of the CMD, a prominent nearly vertical sequence at $2 \lesssim g-i_{\circ} \lesssim 3$ and $19 \lesssim i_{\circ} \lesssim 24$ is visible; the sequence is the result of Galactic disk dwarf stars accumulating over a large range of distances along the line of sight.

The right panel of figure 2 shows the combined CMD of all objects detected in our images and classified as stars in the deep MegaCam field. The CMDs are shown as density contours to reveal features in otherwise crowded regions. In addition to the blue and red sequences of foreground contaminants, a prominent vertical sequence of stars bluer than stars populating the blue foreground sequence, i.e., $(g-i)_{\circ} \leqslant 0$, and covering a wide range of magnitudes, i.e., $19 \lesssim g_{\circ} \lesssim 26$, is present. A second sequence of bright, i.e., $21 \lesssim i_{\circ} \lesssim 22$, and red, i.e., $1.5 \lesssim(g-i)_{\circ} \lesssim 2.5$, stars is present. A third CMD feature is revealed by red giant branch stars.

Figure 3 shows the foreground-subtracted CMD of the stellar population distributed over the MegaCam field. The CMD is typical of stellar systems with extended star formation histories, with a prominent young stellar component. We see the upper main sequence and probable heliumburning blue stars, the red supergiant branch, and probably some young and intermediate age asymptotic giant stars. At $i_{\circ} \sim 25$, the contamination is dominated by background compact galaxies, unresolved in ground-based images and prone to misclassification as stellar objects. Characterising and correcting for the background contamination at faint magnitudes is beyond the scope of the present contribution, and will be addressed in a forthcoming paper. To reduce the contamination from background objects, we have selected stars brighter than $g_{\circ}=25$. A second potential source of contamination when tracing the spatial distribution of young stars in the debris field is the population of intermediate and old stars, either genuine members of the tidal debris or belonging to M81 and/or NGC 3077. These stars start to dominate at $i_{\circ} \sim 24$ and $(g-i)_{\circ} \sim 1$. We have excluded stars fainter than the tip of the red giant branch magnitude at the distance of $\mathrm{M} 81$, and with colours redder than the reddest colours predicted for red supergiant stars. The criteria used to identify young star candidates were defined by inspecting the foreground-corrected CMD and the predicted locations of young stellar populations at the distance of M81. The selection boxes of young stars are shown in Fig. 3. Objects within the blue selection box are defined as main sequence star candidates, and those in the red selection box as red supergiant star candidates. The blue selection box is affected by a modest contamination from foreground Galactic stars and background galaxies, which tend to have red colours, than the red selection box. Despite the selection criteria imposed to select samples of young stars free of background contamination, the red side of the CMD is expected to suffer still from contamination (see Davidge 


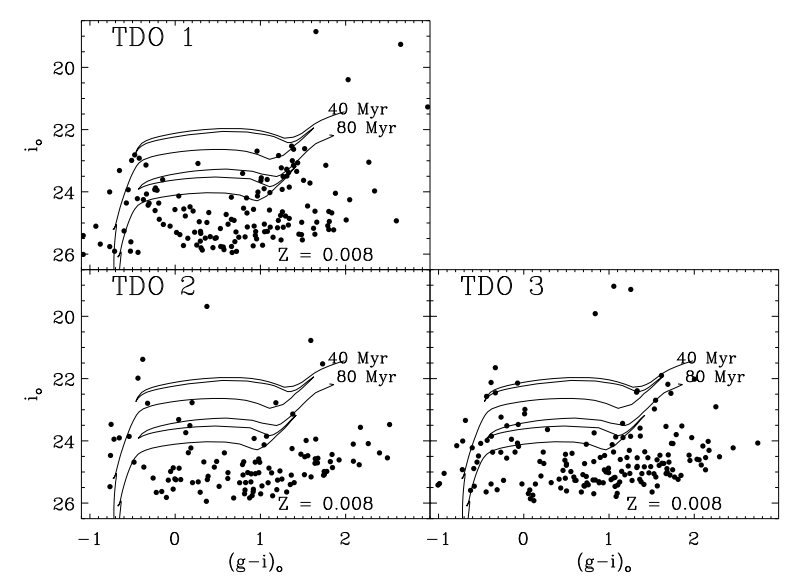

Figure 8. The CMDs of the young stellar grouping identified by Davidge (2008) in the M81 tidal debris. Superimposed on each CMD are the Padova isochrones with a metallicity $\mathrm{Z}=0.008$ and the indicated ages.

(2008) for more details). We have therefore adopted red supergiant star candidates to be only secondary tracers of the debris field.

A potential source of errors when identifying bright stars in distant objects is blending. As discussed in Davidge (2008), who had also used the MegaPrime/MegaCam imager to investigate the bright stellar content of the M81 group, the ages of the young stellar components estimated for Holmberg IX, BK 3N, and Garland from MegaCam CMDs are consistent with those obtained from CMDs obtained with the Hubble Space Telescope (Makarova et al. 2002), suggesting that blending is not severe, if any. The distribution of the young stellar clumps detected in the intergalactic environment along Hi tidal arms rather than been randomly distributed within the field supports this conclusion. By comparing MegaCam images with those obtained with the Advanced Camera for Surveys on board the Hubble Space Telescope, Davidge (2008) had shown that the bright blue sources in the MegaCam images are consistent with being single main sequence stars. In addition, for the blue objects detected in our images to be the results of blending, the blend stars have to be bright in $g$-band yet faint in $i$-band, which is inconsistent with the fact that the bulk of stars in these remote regions of galaxies are intrinsically faint and red.

Figure 4shows the spatial distribution of main sequence star candidates (left panel), and both main sequence and red supergiant candidates (right panel) over the surveyed area. The contours and the grayscale image show the Hi radio map from Yun et al. (1994). The crowding is such that individual stars are not well resolved in the central dense region of M81 and NGC 3077, and their photometry is affected by large errors. As expected, the bulk of young stellar populations are distributed over the star-forming disk of M81. Beyond the young disk of M81, a number of stellar concentrations distributed over a much larger scale are visible, of which a few have been already reported in the literature, i.e., Holmberg IX, BK 3N, Garland, M81-West, Arp's Loop (A0952+69), and the tidal debris objects recently reported by Davidge (2008). In addition to the structure combining M81-West and the first object in the list of Davidge (2008), tracing the continuation of the Hi spiral structure of M81, a second arm of young stars (M81-West2) tracing the continuation of the second Hi spiral structure is visible. Similar structures of young stars tracing the continuation of the Hi spiral structures (M81-SE) are observed to the south along the major axis. Wide field GALEX images of M81 (Gil de Paz et al. 2007) show that these structures of young star candidates are sites of Ultra-Violet emission. More strikingly, a number of previously unknown overdensities of young star candidates can be seen along the tidal bridge of gas stretching from M81 to its neighbour NGC 3077. Interestingly, BK3N and Garland appear to lie at the two extremities of this chain of concentrations of young stars, close to M81 and NGC 3077 respectively. The southern HI arm has been resolved into a series of clouds with sizes of approximatively $4 \mathrm{kpc}$, which are embedded in a more tenuous Hi distribution (van der Hulst 1979). The new young stellar systems to the south of M81 appear to trace the overdense clouds along this Hi arm, with no clear bridge of young stars connecting these systems.

The foreground-corrected CMDs of three control fields sampling similar areas on the sky as the new stellar clumps, and that are located away from the M81-NGC 3077 and M81-M82 debris fields, are shown in Fig.5. The location of the control fields on the sky are shown in Fig.1. The selection boxes of both main sequence and red supergiant star candidates are overplotted on the CMDs of the control fields. The stellar contents of the control fields are all similarly dominated by objects fainter than $g_{\circ} \gtrsim 25$, i.e., red giant branch stars and probable background compact galaxies, with a noticeable absence of both stars with $(g-i)_{\circ} \lesssim 0$, i.e., young main sequence star candidates, and stars with properties similar to those expected for red supergiant stars at the distance of M81, i.e., $(g-i)_{\circ} \sim 1.5$ and $i_{\circ} \sim 22$.

Fig.6] shows the CMDs of the stellar populations that trace the continuations of the Hi both to the north and to the south of the galaxy. Superimposed on the CMD of each stellar system as indicated in each panel are the Padova isochrones with a metallicity $\mathrm{Z}=0.008$ and the indicated ages. The locations of both main sequence stars and red supergiant stars in the CMDs are best matched by $\mathrm{Z}=0.008$ and $\mathrm{Z}=0.004$ isochrones. The plumes of bright main sequence stars are also reasonably matched by isochrones of both higher and lower metallicities, however the predicted locations of red supergiant stars are either bluer or redder than observed.

Fig.7 shows the CMDs of the stellar clumps identified along the southern $\mathrm{HI}$ tidal tail, and the CMDs of BK $3 \mathrm{~N}$ and Garland, both located at the two extremities of the same gaseous arm. Overplotted are the Padova isochrones with a metallicity $\mathrm{Z}=0.008$ and the indicated ages. All the stellar systems were taken to be situated at the same distance as M81. Compared to the control fields, the CMDs of the stellar clumps along the southern Hi tidal tail show clear overdensities of objects with luminosities and colours expected for main sequence and red supergiant star candidates. Similar to the stellar populations tracing the continuations of the Hi spiral structures, the CMDs of stellar systems tracing the southern Hi tail are best matched by isochrones with metallicities $\mathrm{Z} \sim 0.008$. A similar conclusion has been derived for 


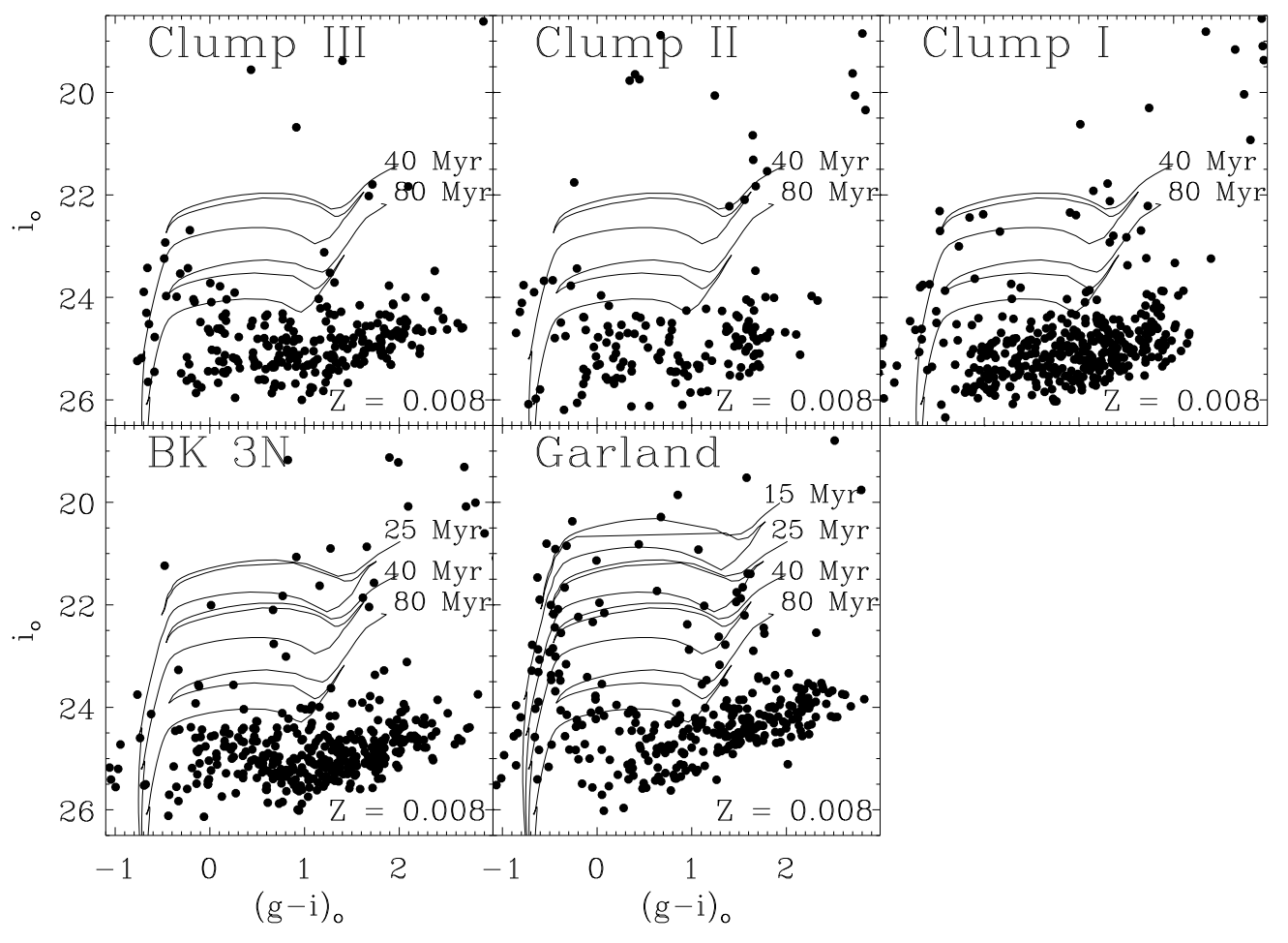

Figure 7. The CMDs of the stellar clumps identified along the southern Hi tidal arm between M81 and NGC 3077, along with BK $3 \mathrm{~N}$ and Garland. Superimposed on each CMD are the Padova isochrones with a metallicity $\mathrm{Z}=0.008$ and the indicated ages.

the young stellar systems in the tidal bridge connecting M81 and M82 (de Mello et al. 2008a), and is consistent with the gas-phase metallicity of an Hi region in Holmberg IX as measured by Makarova et al. (2002).

The CMDs show that the three newly reported stellar systems, similar to BK $3 \mathrm{~N}$ and Garland, contain stars with properties consistent with a range of ages, i.e., they are not simple stellar populations. Davidge (2008) derived a similar conclusion for three other young stellar systems of comparable extents and stellar densities located respectively close to the area identified as M81-West by Sun et al. (2005) and Holmberg IX. The ages of the youngest stars in BK 3N (Garland) are consistent with the absence (the presence) of associated H $\alpha$ emission (Karachentsev \& Kaisin 2007). Stars with photometry that are best matched with isochrones of ages older than a few hundred Myr, or even a few billion years, are present in abundance. We cannot however be sure that these stars are genuine members of the stellar systems, whether they belong to the stellar halo of M81, or whether they were ejected into the intergalactic medium during the interaction.

The CMDs of the stellar structures tracing the continuation of the Hi spiral structures of M81 (M81-West2, M81SE) and Holmberg IX show the presence of stars younger than these in the stellar over-densities distributed along the southern HI arm. The CMDs of the new stellar clumps show the presence of stars with photometric properties consistent with their youngest stars formed around $40 \mathrm{Myr}$ ago. BK $3 \mathrm{~N}$, located at the western end of the Hi tidal arm, appears to be dominated by stars of similar ages to the other three clumps along the tidal arm. The Garland structure, at the easter end of the Hi tidal arm, however contains stars as young as those observed in Holmberg IX and the spiral arms. This suggests that the star formation activity was likely truncated earlier in the stellar systems within the tidal field that are away from large galaxies than in those in their close vicinity.

The $i_{\circ}$ vs. $(g-i)$ 。CMDs of the recently reported stellar groupings in the debris field of M81 by Davidge (2008) are shown in Fig.8. The locations of these stellar groupings on the sky are indicated in Fig.1 Superimposed on the CMD of each stellar system as indicated in each panel are the Padova isochrones with a metallicity $\mathrm{Z}=0.008$ and the indicated ages. Similar to other young stellar systems within the debris field of M81, the CMDs of those stellar systems are best matched by isochrones with metallicities $\mathrm{Z} \sim 0.008$. The photometric properties of turn-off stars in those groupings are consistent with their youngest stars formed $\sim 40 \mathrm{Myr}$ ago, consistent with the age estimates of Davidge (2008). These stellar systems appear to be dominated by stellar populations similar to those of the stellar clumps distributed along the southern Hi arm.

\section{DISCUSSION}

Numerous multi-wavelength data sets have unambiguously identified localised regions with signs of current and/or recent star formation activity distributed along Hi tidal tails in interacting systems (e.g. Weilbacher et al. 2003; 
Hibbard et al. 2005; de Mello et al. 2008b), with a number of these regions suspected to be bounded, the so-called tidal dwarf galaxies (e.g. Duc \& Mirrabel 1998; Braine et al. 2001; Hancock et al. 2009). The star formation activity along the tidal tails appears to be distributed with a similar morphology to Hi (e.g. Hibbard et al. 2005; Neff et al. 2005; Hancock et al. 2007), in agreement with our finding of the new stellar clumps reported here tracing the dense regions along the southern Hi tidal tail. Hibbard et al. (2005) have found that UV colours of localised regions of star formation along the tidal tails of the archetypal merging system NGC 4038/39 are consistent with continuing star formation. Neff et al. (2005) have argued however, for the case of NGC $7769 / 71$, NGC 5713/19, and the NGC 520 system, that most of young stars in the tails have most likely formed in single bursts. The CMDs of stellar clumps in the debris field of M81, e.g. Arp's loop region, Holmberg IX, contain stars with photometric properties consistent with a wide range of ages, i.e., from $\sim 10 \mathrm{Myr}$ to $\sim 1 \mathrm{Gyr}$ (e.g. de Mello et al. 2008b; Sabbi et al. 2008), suggesting extended star formation histories. Deep imaging data show however a lack of any concentration of old stars associated with the blue stars, suggesting that the "old" stellar component seen in the CMDs of those stellar clumps were formed in the stellar disks of M81 and ejected into the intergalactic medium during tidal passages, whereas the young stars have formed in the tidal debris (de Mello et al. 2008b; Weisz et al. 2008). The spatial distribution of red giant branch stars over the region connecting M81 and NGC 3077 does not show any noticeable concentrations of old stars associated to the young stellar clumps identified along the southern Hi tidal tail. As for, e.g. Holmborg IX and Arp's loop region, this suggests that the old stars seen in the CMDs of the newly reported stellar clumps should have come from one of the interacting systems while, since the stellar clumps along the southern tidal tail are considerably younger than its dynamical age, e.g. $\sim 250$ Myr (Yun 1999), young stars formed on site.

Fitting single stellar population synthesis models to UV/optical colours of UV-bright stellar substructures within the tidal tails of four ongoing galaxy mergers, Neff et al. (2005) found that the star formation appears to be older near the parent galaxies and younger at increasing distances. They have suggested that this could be because the star formation occurs progressively along the tails, or because the star formation has been inhibited near the galaxy/tail interface. Hibbard et al. (2005) had reported negative UV and optical colour gradients along the tidal tails of the "Antennae" system, indicative of negative age gradients when moving outward along the tails (see also Hibbard et al. 2001). Note that the observed colour gradients could be accounted for alternatively by the presence of composite stellar populations. The star formation within the tidal tails in M81 debris field appears to be different. The CMDs of the bulk of stellar clumps in the debris field of M81 indicate that they have ceased forming stars at similar epochs in the past, i.e., $\sim 40 \mathrm{Myr}$ ago. This suggests that the star formation throughout M81 tidal debris field could have been triggered by common events (see Davidge 2008, for a similar conclusion), and that the physical conditions within the dense regions along the southern Hi tidal tail are comparable. The systems within the debris field with younger stars, i.e., Holmberg IX and Garland, are both in the close vicin-
Table 1. Coordinates of the new young stellar clumps along the southern Hi arm.

\begin{tabular}{lll}
\hline ID & R.A. (J2000) & Dec. (J2000) \\
\hline Clump I & $09: 57: 21.2$ & $68: 42: 55$ \\
Clump II & $09: 59: 40.4$ & $68: 39: 19$ \\
Clump III & $10: 00: 40.4$ & $68: 39: 37$
\end{tabular}

ity of M81 and NGC 3077 respectively, in contrast with the findings of Neff et al. (2005). The diversity of these star formation histories could be most likely related to different gas contents and conditions within the tidal tails of those interacting systems.

The exact nature of the previously known young stellar systems within the tidal field of the M81 group, i.e., Holmberg IX, Gerland, and BK 3N, is not entirely clear yet. Detailed modelling of the dynamics of the M81 group suggests that the three largest galaxies in the system had an interaction $\sim 250 \mathrm{Myr}$ ago for M81 and NGC 3077, and 200 Myr ago for M82 and M81 (Yun 1999). The modelling of optical CMDs of Holmberg IX, BK 3N, and Arp's loop, of comparable depth to ones presented here, suggested that these galaxies have experienced star formation between about 20 and 200 Myr ago (Makarova et al. 2002). It has been argued then that these galaxies are tidal dwarf condidates that formed from dust and gas that was blown away from M81 and/or other galaxies in the group (Boyce et al. 2001; Makarova et al. 2002). BK3N may be alternatively a pre-existing dwarf irregular galaxy undergoing an interaction with M81 (Boyce et al. 2001). The old stellar population associated spatially to Holmberg IX is suspected to belong quite likely to the outer regions of M81, suggesting that this stellar system is of a tidal origin (Sabbi et al. 2008).

The ages and metallicities of the isochrones that best reproduce the CMDs of the stellar systems along the southern Hi arm are similar to these needed to account for the properties of the stellar contents of previously known tidal dwarf candidates in the tidal debris field. This suggests that they both could share similar star formation histories and might be associated to similar events. These isochrone metallicities are significantly higher than the typical metallicities of dwarf galaxies of comparable luminosities (e.g. Richer \& McCall 1995). This suggests that these stellar systems have been assembled from pre-enriched material. This is consistent with the conclusions of Boone et al. (2005) who found that the abundances and physical conditions of the molecular complex situated near the line of sight toward Holmberg IX are similar to those found in the disks of spiral galaxies. The distribution of the stellar clumps along an Hi tail, tracing the densest clouds within the gaseous arm, indicates that these systems may have been assembled out of gas pulled from one of the large interacting galaxies in the group. A primary criterion for the determination of the nature of these objects is to measure their mass-to-light ratio, which tend to be low for tidal dwarf galaxies due to the absence of dark matter (e.g. Barnes \& Hernquist 1992; Duc et al. 2000). Unfortunately this cannot be measured from the dataset in hand. Without this measurement, we can only conclude that the newly reported stellar clumps are likely (among the nearest) tidal dwarf galaxies. 


\section{ACKNOWLEDGMENTS}

We would like to warmly thank Mike Irwin for (various) helpful discussions.

\section{REFERENCES}

Appleton P. N., Davies R. D, Stephenson R. J, 1981, MNRAS, 195, 327

Barnes J. E., Hernquist L., Nature, 360, 715

Boone F., et al., 2005, A\&A, 429, 129

Boyce P. J, et al., 2001, ApJ, 560, 127

Braine J., Duc P.-A., Lisenfeld U., Charmandaris V.,

Vallejo O., Leon S., Brinks E., A\&A, 378, 51

Brown T. M., et al. 2006, ApJ, 652, 323

Chiboucas K., Karachentsev I. D, Tully B. R., 2008, AJ, in press (astroph/08051250)

Davidge T. J, 2008, PASP, 120, 1145

de Mello D. F., Smith L. J., Sabbi E., Gallagher J. S., Mountain M., Harbeck D. R., 2008, AJ, 135, 548

de Mello D. F., Torres-Flores S., Mendes de Oliveira C., 2008, AJ, 135, 319

Duc P.-A., Mirabel I. F., 1998, A\&A, 333, 813

Duc P.-A., Brinks E., Spingel V., Pichardo B., Weilbacher P., Mirabel I. F., 2000, AJ, 120, 1238

Durrell P. R., Decesar M. E., Ciardullo R., Hurley-Keller D., Feldmeier J. J., 2004, IAU Symposium no. 217, Edited by P.-A. Duc, J. Braine, and E. Brinks. San Francisco: Astronomical Society of the Pacific, p.90.

Freedman W. L., et al., 1994, ApJ, 427, 628

Gil de Paz A., et al., 2007, ApJS, 173, 185

Hammer F., Puech M., Chemin L., FLores H., Lehnert M. D., 2007, ApJ, 662, 322

Hancock M., Smith B. J., Struck C., Giroux M. L., Appleton P. N., Charmandaris V., Reach W. T., 2007, AJ, 133, 676

Hancock M., Smith B. J., Struck C., Giroux M. L., Hurlock S., 2009, AJ, in press

Hibbard J. E., et al., 2005, 619, L87

Hibbard J. E., van der Hulst J. M., Barnes J. E., Rich R. M. 2001, AJ, 122, 2969

Ibata R., Chapman S., Ferguson A. M. N., Lewis G. F., Irwin M., Tanvir N., 2005, ApJ, 634, 287

Ibata R., Martin N. F., Irwin M., Chapman S., Ferguson A. M. N., Lewis G. F., McConnachie A. W., 2007, ApJ, Irwin M., \& Lewis J., 2001, NewAR, 45, 105

Karachentsev I. D., Karachentseva V. E., Boerngen F., 1985, MNRAS, 217, 731

Karachentsev I. D., et al., 2001, A\&A, 375, 359

Karachentsev I. D., \& Kaisin S. S., 2007, AJ, 133, 1883

Makarova L. N, et al.,2002, A\&A, 396, 473

Neff S. G., et al., 2005, ApJ, 619, 91L

Renda A., Gibson B.K., Mouhcine M., Ibata R., Kawata D., Flynn C., Brook C. B., MNRAS, 2005, 363, 16

Richer M. G., McCall M. L., 1995, ApJ, 445, 642

Robin A., Reylé C., Derrière S., \& Picaud S., 2003, A\&A, 409, 523

Sabbi E., Gallagher J. S., Smith L. J., de Mello D. F., Mountain M., 2008, ApJ, 676, L113

Schlegel D. J., Finkbeiner D. P., Davis M., 1998, ApJ, 500, 525
Ségall M., Ibata R., Irwin M. J., Martin N., Chapman S., 2007, MNRAS, 375, 831

Sun W.-H., et al., 2005, ApJ, 630, L133

Toomre A., Toomre J., 1972, ApJ, 178, 623

Weisz D. R., Skillman E. D., Cannon J. M., Dolphin A. E., Kennicutt R. C., Lee J., Walter F., 2008, ApJ, 689, 160 van der Hulst J. M., 1979, A\&A, 75, 97

Weilbacher P. M., Duc P.-A., Fritze-v. Alvensleben U., 2003, A\&A, 397, 545

Yun M. S.,Ho P. T. P., Lo K. Y., 1994, Nature, 372, 530

Yun M. S, 1999, Galaxy interaction at low and high redshift (IAU Symp. 186), Ed. J. E. Barnes \& D. B. Sanders, 81

This paper has been typeset from a $\mathrm{T}_{\mathrm{E}} \mathrm{X} / \mathrm{L}^{\mathrm{A}} \mathrm{T}_{\mathrm{E}} \mathrm{X}$ file prepared by the author. 


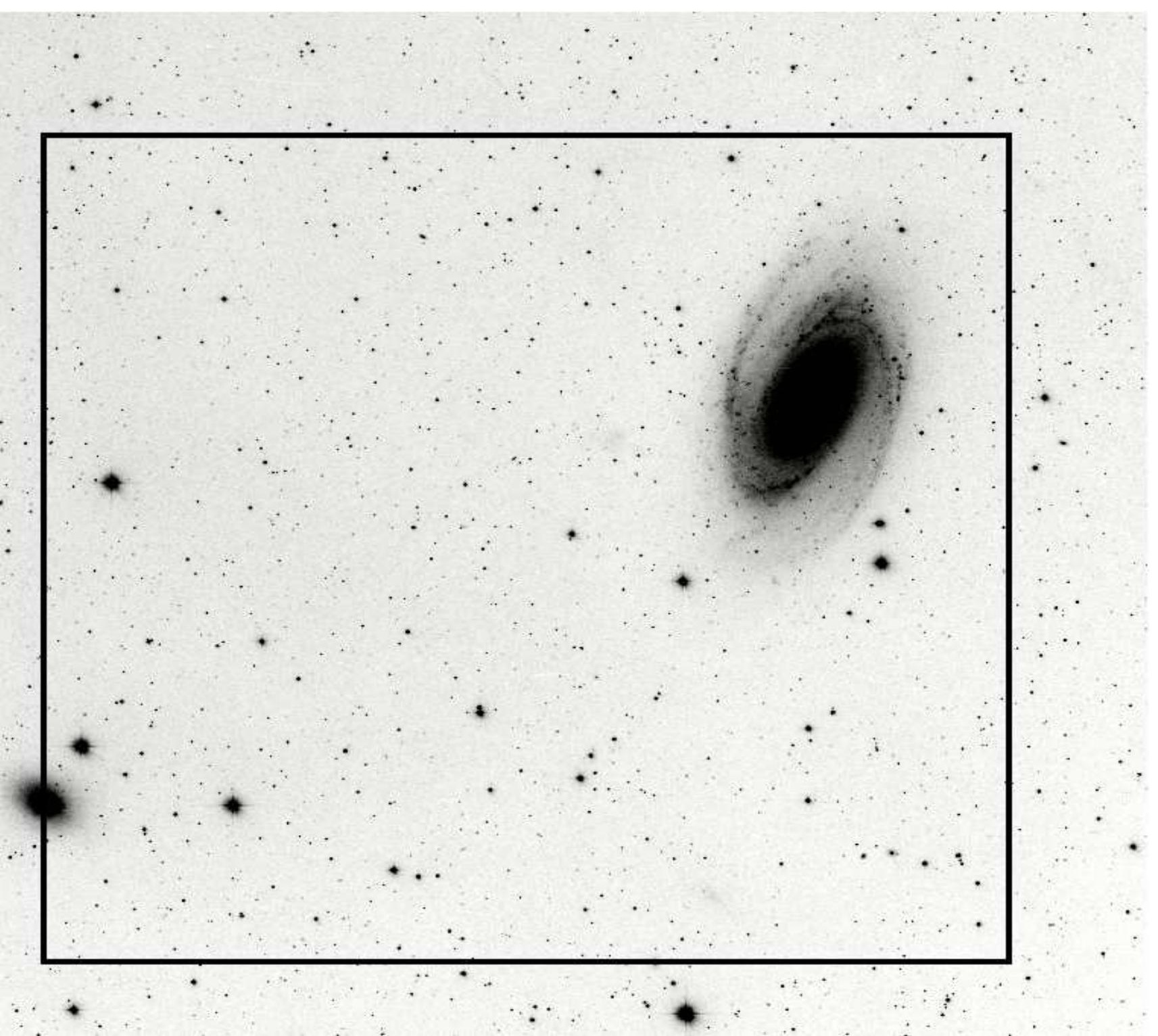




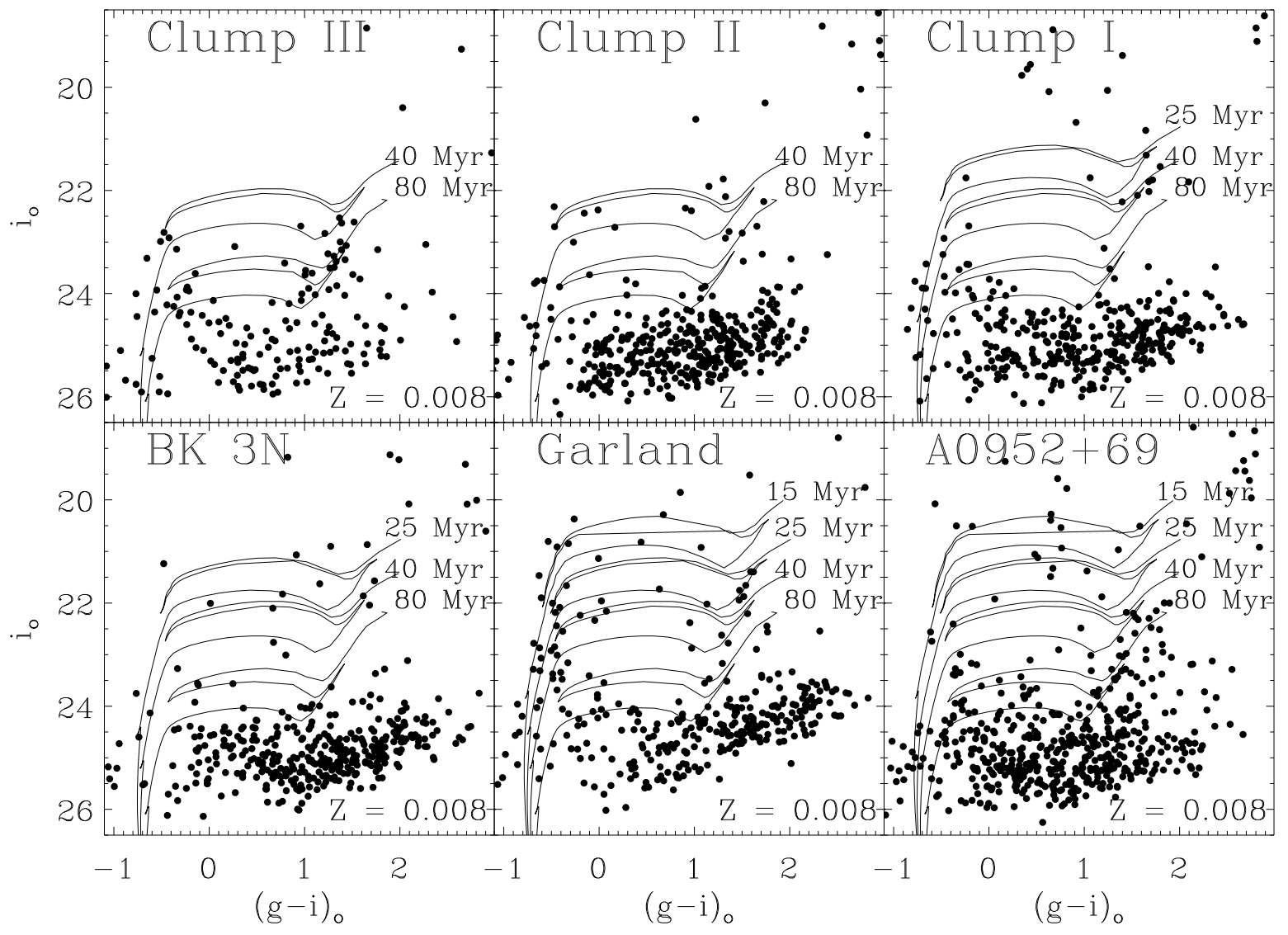




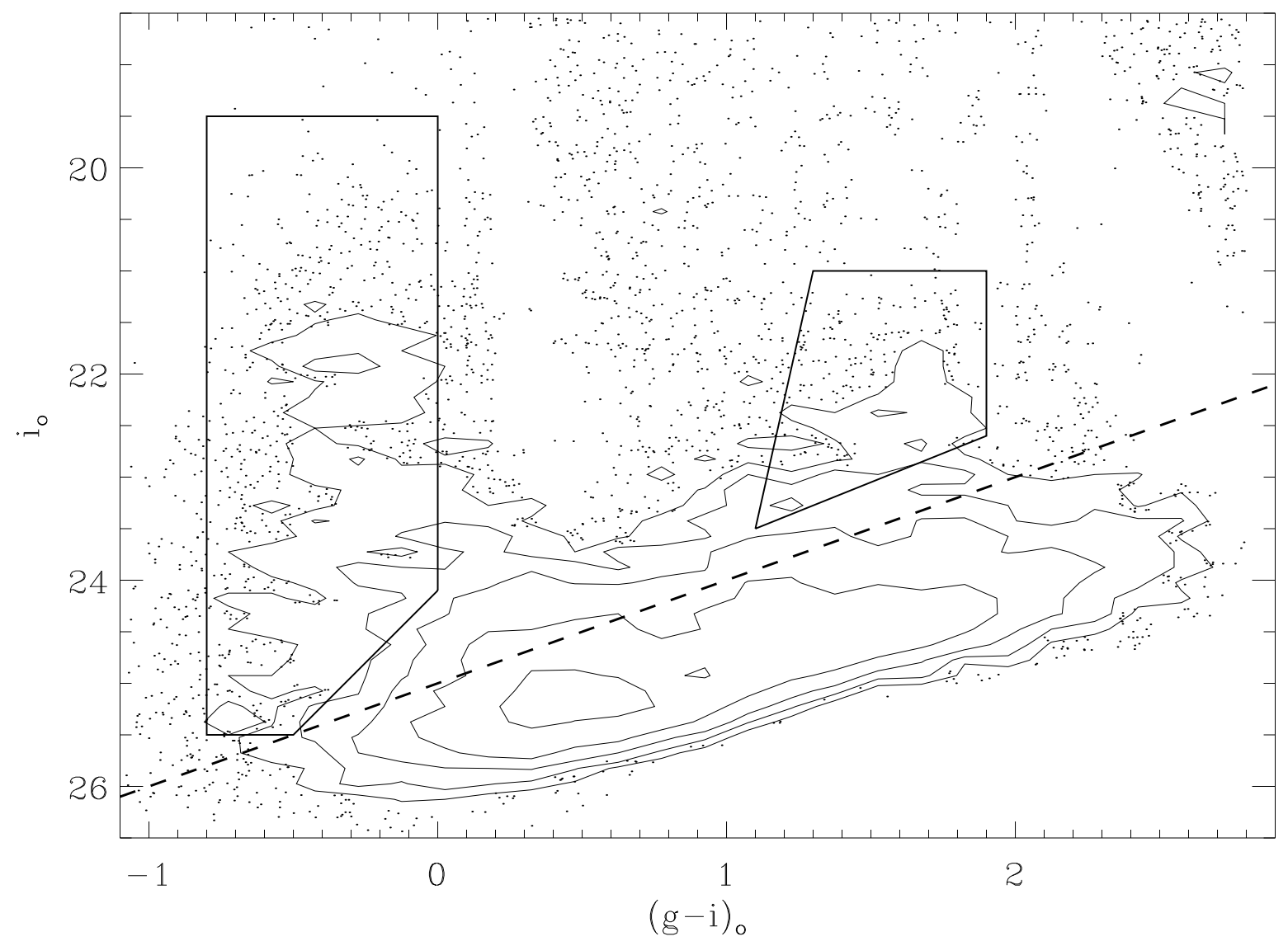




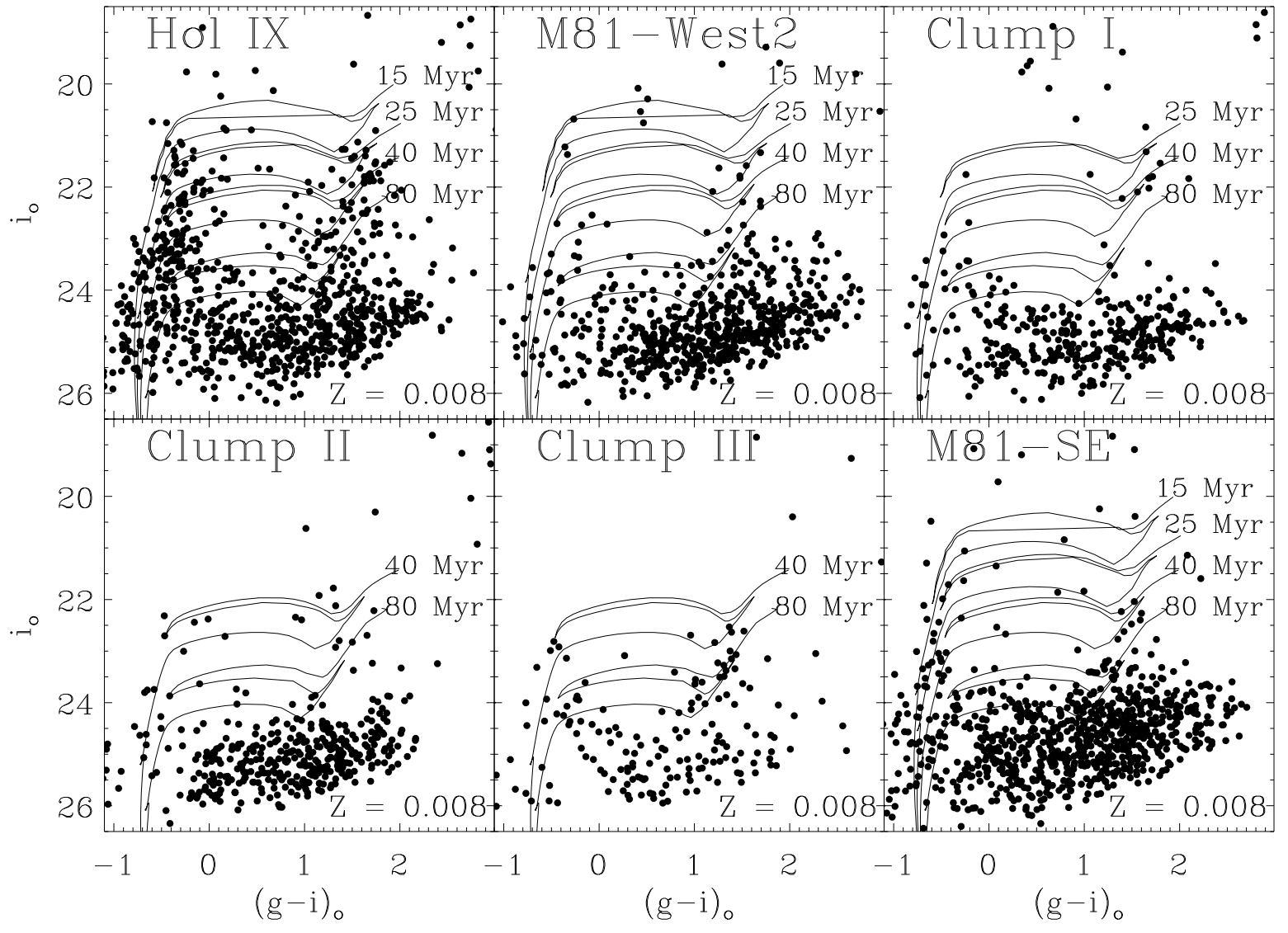

